Abstract S107 Table 1

\begin{tabular}{|c|c|c|c|c|c|c|}
\hline Variable & $\begin{array}{l}\text { Exacerbation Start } \\
\text { (No viral symptoms) } n=23\end{array}$ & $\begin{array}{l}\text { End Exacerbation } \\
\text { (No viral symptoms) } n=23\end{array}$ & p Value & $\begin{array}{l}\text { Start Exacerbation } \\
\text { (Viral symptoms) } n=17\end{array}$ & $\begin{array}{l}\text { End Exacerbation } \\
\text { (Viral symptoms) } n=17\end{array}$ & p Value \\
\hline $24 \mathrm{~h}$ sputum volume (ml) & $15(10-15)$ & $3(0-10)$ & $<0.0001$ & $20(10-20)$ & $5(2-8)$ & $<0.0001$ \\
\hline$\%(\mathrm{n})$ purulent sputum & $95.6(22)$ & $26.1(6)$ & $<0.0001$ & $82.3(14)$ & $0(0)$ & $<0.0001$ \\
\hline ISWT (m) & $210(80-350)$ & $350(140-430)$ & $<0.0001$ & $300(100-410)$ & $380(255-585)$ & $<0.0001$ \\
\hline $\mathrm{ESR}(\mathrm{mm} / \mathrm{hr})$ & $26(14-44)$ & $18(9-41)$ & 0.04 & $14(8.5-25)$ & $12(7.5-14)$ & 0.01 \\
\hline $\operatorname{CRP}(\mathrm{mg} / \mathrm{l})$ & $14(10-33)$ & $5(2-15)$ & $<0.0001$ & $14(8-26.5)$ & $5(2-8)$ & 0.002 \\
\hline WCC $\left(\times 10^{9}\right)$ (range $\left.4-11\right)$ & $9.3(7.3-12)$ & $8.6(7.2-9.7)$ & 0.005 & $8(6-12.2)$ & $7.1(5.4-8.75)$ & 0.04 \\
\hline $\begin{array}{l}\text { Neutrophil count }\left(\times 10^{9}\right) \\
\text { (range } 2-7.5)\end{array}$ & $6.42(5.5-8.41)$ & $5.22(4.64-6.76)$ & 0.02 & $5.44(3.5-8.86)$ & $3.85(3.06-6.2)$ & 0.07 \\
\hline $\begin{array}{l}\text { Lymphocyte count }\left(\times 10^{9}\right) \\
\text { (range } 1.5-4)\end{array}$ & $1.76(1.08-2.18)$ & $1.71(1.32-2.13)$ & 0.55 & $1.82(1.41-2.63)$ & $1.76(1.52-2.16)$ & 0.8 \\
\hline $\begin{array}{l}\text { Pathogens isolated } \\
\text { from sputum \% (n) }\end{array}$ & $100(23)$ & $30.4(7)$ & 0.009 & $100(17)$ & $23.5(4)$ & $<0.0001$ \\
\hline
\end{tabular}

CRP, C reactive protein; ESR, Erythrocyte Sedimentation Rate; WCC, White cell count

bacteriology; sputum colour; 24 h sputum volume; respiratory viral PCR [Influenza A and B; Respiratory Syncytial Virus; Parainfluenza Type 1, 2, 3; Adenovirus]; incremental shuttle walk test; total white cell count; lymphocyte count; neutrophil count; $C$ reactive protein; Erythrocyte Sedimentation Rate. Data is presented as median (IOR) and groups compared using the Mann-Whitney U Test.

Results 40 patients were included. 17 reported viral symptoms. Of the 17 patients who reported viral symptoms, only 2 had positive viral PCR [Influenza Type B $(n=1)$ and Respiratory Syncytial Virus $(n=1)]$. No patient in the group not reporting viral symptoms had a positive viral PCR. At the start of the exacerbation, there was no significant difference in any parameter between the groups and both groups had a similar and positive impact with 2-week course of antibiotic therapy. Abstract S107 table 1 details the measurements at the start and end of the exacerbation for each group.

Conclusion There was a low prevalence of positive virology despite the presence of viral symptoms and outcomes were similar in patients with and without viral symptoms. Larger cohort studies are needed.

\section{S108 EXPERT CONSENSUS ON DIAGNOSTIC CRITERIA AND TERTIARY SERVICE REQUIREMENTS FOR BRONCHIECTASIS}

doi:10.1136/thoraxjnl-2011-201054b.108

${ }^{1} \mathrm{~J}$ Holme, ${ }^{2} \mathrm{~S}$ Bianchi, ${ }^{3} \mathrm{I}$ Clifton, ${ }^{4} \mathrm{~A}$ De-Soyza, ${ }^{2} \mathrm{~F}$ Edenborough, ${ }^{3} \mathrm{D}$ Peckham, ${ }^{2} \mathrm{O}$ Pirzada, ${ }^{5} \mathrm{P}$ Walker, ${ }^{6} \mathrm{M}$ Walshaw, ${ }^{1} \mathrm{R}$ Niven. ${ }^{1}$ University Hospital of South Manchester NHS Foundation Trust, Manchester, UK; ${ }^{2}$ Sheffield Teaching Hospitals NHS Foundation Trust, Sheffield, UK; ${ }^{3}$ Leeds Teaching Hospitals NHS Trust, Leeds, UK; ${ }^{4}$ Newcastle Hospitals NHS Foundation Trust, Newcastle, UK; ${ }^{5}$ Aintree University Hospitals NHS Foundation Trust, Liverpool, UK; ${ }^{6}$ Liverpool Heart and Chest Hospital NHS Foundation Trust, Liverpool, UK

Introduction There are no agreed diagnostic criteria for bronchiectasis and no stated minimum requirements for a tertiary service. The Northern Bronchiectasis Group aimed to use the validated RAND technique to form consensus opinions on these issues. Method Following literature review, a questionnaire was devised containing 89 statements relevant to the topics above. Eight expert members rated their level of agreement with the statements from 1 (not relevant) to 9 (mandatory). Following a group debate about these statements, the experts re-structured some statements then re-rated the questionnaire. Consensus agreement, indifference or disagreement was reached if $7 / 8$ members' scores were in the 7-9, 4-6 or 1-3 ranges respectively.

Results There was consensus agreement for 31/89, consensus indifference for $5 / 89$ and consensus disagreement for $12 / 89$ state- ments. Consensus was not reached for 41/89 statements. It was agreed daily sputum production would prompt investigation for bronchiectasis, CT was always necessary, and the following factors, support the diagnosis: bronchoarterial ratio $>1.0$, non-tapering bronchi, thickened airway walls, irreversible changes. It was agreed that a tertiary service should provide: access to HRCT, spirometry, routine and fungal sputum cultures, ciliary function testing; functional antibodies and immunoglobulins for all, antibiotic \& hypertonic saline nebuliser challenges, nebuliser loan and maintenance, home iv antibiotic service (preferably by patients in their own homes), portacath insertion, physiotherapy at least annually, access to a dietician, immunologist, microbiologist with an interest in bronchiectasis and pulmonary rehabilitation. Specialist nurses could see selected patients and separate clinics are desirable for patients colonised with pseudomonas. There was indifference to the availability of telephone consultations, posted sputum analysis, iv antibiotic service based in the community (not in patients' home), a patient support group and patient educational sessions. Consensus was not reached regarding if respiratory infection, bronchoarterial ratios of $>1.5$ or $>2.0$ or abnormal spirometry are necessary to diagnose bronchiectasis; or if a consultant should see patients at most visits, iv antibiotics could be given by a nurse in the patients home and if access to palliative care was necessary in a tertiary service.

Conclusion Comprehensive consensus statements regarding the diagnostic criteria for bronchiectasis and tertiary service requirements have been formed.

\section{ILD mechanisms}

\section{S109 CONTRIBUTION OF ABERRANT MONOCYTE-NATURAL KILLER T (NKT) CELL AXIS TO IMMUNE-PATHOLOGY IN SARCOIDOSIS}

doi:10.1136/thoraxjnl-2011-201054b.109

A P Crawshaw, W L Kok, L Denney, L-P Ho. Human Immunology Unit, Weatherall Institute of Molecular Medicine, University of Oxford, Oxford, UK

Introduction Sarcoidosis is a multisystem disorder characterisedby an overactive CD4 (T-helper 1) cell response to an undefined antigen, macrophage activation and granuloma formation. It has also been shown that monocytes (precursors to macrophages) are increased in sarcoidosis. We have shown that NKT cells, a specialised subset of immuneregulatory $\mathrm{T}$ cells, are deficient in sarcoidosis, and that in NKT knock-out mice, monocytes accumulate at sites of inflammation in both models of influenza A infection and multiple sclerosis. Here, we hypothesise that NKT cells control monocyte function and 
that NKT cell deficiency in sarcoidosis results in abnormal monocyte activity.

Methods Twenty-five steroid-naïve non-smoking patients with histological confirmation of sarcoidosis were recruited from the Sarcoidosis-ILD service. Circulating monocyte numbers and phenotype were first characterised using multi-colour flow cytometry. We then isolated monocytes from blood using magnetic microbeads, examined cytokine production after LPS stimulation with intracellular cytokine FACS staining and ELISA; and using monocyte-NKT cell co-culture assays, questioned whether NKT cells affected these monocytic functions.

Results We found an increase in circulating CD14CD16 inflammatory monocytes in patients with sarcoidosis, and identified a population of interleukin 10 producing monocytes in patients and controls after LPS stimulation. Monocytes from sarcoidosis patients have reduced capacity to produce IL-10 after LPS stimulation compared to control $(6.37 \%$ vs $11.71 \%$ of total monocytes, $\mathrm{p}<0.001$, Abstract S109 figure 1A); but addition of NKT cells improved this capacity $(6.37 \%$ to $9.13 \%, p<0.001$, Abstract S109 figure $1 \mathrm{~B})$. We then questioned the role of IL10-producing monocytes and show (with mixed lymphocyte reaction and CFSE assays) that these cells suppress $T$ cell proliferation ( $p<0.001$, Abstract S109 figure 1C).

Conclusions Our data show that sarcoidosis patients have increased inflammatory monocytes but a reduced IL-10-producing, $\mathrm{T}$ cell suppressing subset. NKTcells were able to interact with monocytes in vitro and increased IL-10 production by monocytes. These previously unrecognised findings, both in monocyte-NKT cross talk and in sarcoidosis immunobiology, suggest that one consequence of NKT deficiency in sarcoidosis is abnormal monocyte function with resultant loss in control of $\mathrm{T}$ cell proliferation. This reveals a potential new pathway of pathogenesis in sarcoidosis.
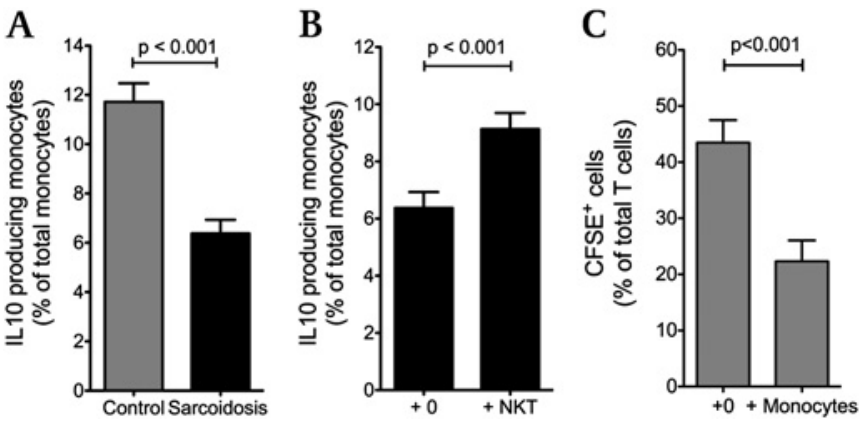

Abstract S109 Figure 1

\section{S110 TARGETED DELETION OF G $\alpha 0 / \mathbf{G} \alpha 11$ IN SURFACTANT PROTEIN C-POSITIVE EPITHELIAL CELLS REDUCES TGFß ACTIVATION AND RESULTS IN INFLAMMATION AND ALVEOLAR AIRSPACE ENLARGEMENT}

doi:10.1136/thoraxjnl-2011-201054b.110

${ }^{1} \mathrm{~A}$ E John, ${ }^{1} \mathrm{~A}$ Habgood, ${ }^{1} \mathrm{~J}$ Porte, ${ }^{1} \mathrm{~A}$ Tatler, ${ }^{1} \mathrm{~L}$ Jolly, ${ }^{2} \mathrm{~S}$ Offermanns, ${ }^{1} \mathrm{R}$ G Jenkins. ${ }^{1}$ University of Nottingham, Nottingham, UK; ${ }^{2}$ Max-Planck-Institute for Heart and Lung Research, Bad Nauheim, Germany

Activation of latent TGFß by the epithelially-restricted $\alpha v ß 6$ integrin is critical in the pathogenesis of lung injury and fibrosis, and disruption of this pathway promotes emphysema development. We have previously shown that Gaq and RhoA signalling pathways are central to avb6 integrin induced TGFß activation in vitro. To assess the role of the $\mathrm{Gq} / 11$ signalling pathway in the lungs, we generated mice with deletion of the Gq and G11 $\alpha$-subunits in Surfactant protein C (SftpC)-positive epithelial cells (Gq/11DKO). SftpC-Cre mice were crossed with constitutive Ga11-deficient animals $\left(\mathrm{Gna11}^{-1-}\right.$; G11KO) carrying floxed alleles of the Gaq gene (Gnaq $q^{f / f l}$ ) and then backcrossed onto appropriate null mice. Lungs were perfused, inflated and fixed prior to processing for histological and immunohistochemical analysis at 2, 4, 6 and 8 weeks. Bronchoalveolar lavage (BAL) cells were collected at 6 weeks for mRNA, nuclear protein extraction or histological analysis. Focal inflammatory infiltrates were visible in the Gq/11DKO lungs as early as 2 weeks, but became larger and more widespread at later timepoints. $\mathrm{Gq} / 11 \mathrm{DKO}$ mice also exhibited significant age-related airspace enlargement compared with G11KO mice from 4 weeks onwards. From 6 weeks, inflammation was closely associated with localised disruption of the alveolar architecture and the appearance of enlarged and vacuolated macrophages within the airspaces. BAL fluid from $\mathrm{Gq} / 11 \mathrm{DKO}$ mice contained significantly higher cells numbers $\left(12.5 \pm 2.5 \times 10^{5}\right)$ than G11KO mice $\left(0.96 \pm 0.2 \times 10^{5}\right)$ with increases in the percentage of neutrophils, lymphocytes and enlarged and vacuolated alveolar macrophages. mRNA analysis of $\mathrm{Gq} / 11 \mathrm{DKO}$ BAL cells showed significantly increased MMP12, RELM $\alpha$ and Arginase 1 suggesting an increase in the number of alternatively activated macrophages. To assess levels of active TGFß in the lungs, phosphorylated SMAD2 (pSMAD2), a component of the TGFß signalling pathway, was measured by ELISA of nuclear extracts from BAL cells. Gq/11DKO BAL cells contained significantly lower levels of pSMAD2 than those from G11KO mice, suggesting decreased levels of active TGFß in the lungs of Gq/11DKO mice. These data suggest that the Gaq/11 signalling pathway in SftpC-positive epithelial cells regulates TGF $\beta$ activation in the lungs and that deficiency in this pathway results in pulmonary inflammation and disruption of the alveolar architecture of the lung.

\section{S111 THE ROLE OF TNF-RELATED APOPTOSIS INDUCING LIGAND (TRAIL) IN PULMONARY FIBROSIS}

doi:10.1136/thoraxjnl-2011-201054b.111

${ }^{1} \mathrm{E}$ E McGrath, ${ }^{1} \mathrm{~A}$ Lawrie, ${ }^{1} \mathrm{H}$ Marriott, ${ }^{2} \mathrm{P} F$ Mercer, ${ }^{1} \mathrm{~S} S$ Cross, ${ }^{2} \mathrm{R} \mathrm{C}$ Chambers, ${ }^{1} \mathrm{D}$ H Dockrell, 'M K B Whyte. 'University of Sheffield, Sheffield, UK; ${ }^{2}$ University College London, London, UK

Introduction The concept of driving cellular apoptosis as a potential therapy for diseases characterised by inappropriate cellular persistence or proliferation is of widespread interest. We previously showed a death receptor ligand, TRAIL, accelerates neutrophil apoptosis without associated cell activation $(J$ Immunol 170:1027-33) and other work revealed TRAIL-induced apoptosis of human lung fibroblasts. The aims of this project were to study the role of TRAIL in a bleomycin lung injury model in wild-type and TRAIL-/- mice and in patients with idiopathic pulmonary fibrosis (IPF).

Methods Mice received intratracheal bleomycin or saline control. Bronchoalveolar lavage (BAL) at 3, 7, 16 and 23 days was analysed by cytospin morphology and haemocytometer count for \% neutrophils, \% neutrophil apoptosis, total number of neutrophils and total number of apoptotic cells. Flow cytometry was also used to analyse apoptosis. Collagen deposition in whole lung samples was analysed using a hydroxyproline assay. TRAIL expression and TUNEL positive events were also analysed. Serum and lung tissue from IPF patients/controls were examined for TRAIL expression and concentration. Lung function and survival data were retrieved from patient charts.

Results BAL analysis revealed statistically significant differences between TRAIL-/- and wild-type mice, with TRAIL-/- mice showing increased neutrophil numbers and reduced neutrophil apoptosis as absolute count or as \% total cell count. Collagen deposition was statistically greater in TRAIL-/- mice at 16 days. At day 23, TRAIL-/- mice had decreased TUNEL positive events compared to wild-type mice. Histological analysis of murine lung sections revealed specific TRAIL expression in bronchus associated 